\title{
Benefits and Harms of Electrical Neuromodulation for Chronic Pelvic Pain: A Systematic Review
}

\author{
a, *, Angela M. Cottrell b, Marc P. Schneider c, Sanchia Goonewardene d, \\ ${ }_{6}$ Andrew P. Baranowski e, Daniel E. Engeler f, Jan Borovicka f, Paulo Dinis-Oliveira g, Suzy Elneil h, \\ ${ }_{7}$ John Hughes i, Bert E.J. Messelink j, Amanda C. de C Q7 Williamsk \\ 8 a Assistant Guidelines Office, European Association of Urology, Arnhem, The Q2 Netherlands ; b Royal Devon and Exeter Hospital (Wonford), Exeter, UK \\ ¿University of Bern, Bern, Switzerland; d East of England Deanery, London, UK; e Guy's Hospital, London, UK; f Kantonsspital St. Gallen, St. Gallen, \\ Switzerland; g University of Porto, Porto, Portugal; h University College Hospital and the National Hospital for Neurology and Neurosurgery, London, UK; i The \\ James Cook University Hospital, Middlesbrough, UK; j Faculty of Medical Sciences, Urology, University of Groningen, Groningen, The Netherlands; $k$ \\ University College London, London, UK \\ * Corresponding author.
}

\section{Review - Pelvic Pain TAKE HOME MESSAGE}

Benefits and Harms of Electrical Neuromodulation for Chronic Pelvic Pain: A Systematic Review

A.M. Cottrell, M.P. Schneider, S. Goonewardene, A.P. Baranowski, D.E. Engeler, J. Borovicka, P. Dinis-Oliveira, S. Elneil, J. Hughes, B.E.J. Messelink, A.C. de C Williams

Chronic pelvic pain (CPP) is chronic or persistent pain perceived in structures related to the pelvis of men and women. These individuals may suffer significant distress and detriment to their daily living and quality of life. Neuromodulation may provide an effective treatment option in patients with CPP refractory to standard treatment, reducing pain and improving quality of life with an acceptable rate of complications. However, study quality is insufficient for a more certain conclusion, and therefore larger-scale, well-designed, and powered randomized controlled trials with long-term outcomes are needed. https://doi.org/10.1016/j.euf.2019.09.011

https://doi.org/10.1016/j.euf.2019.09.011

Accepted September 17, 2019

Keywords:

Chronic pelvic pain

Neuromodulation

Pain relief

Percutaneous tibial nerve stimulation

Transcutaneous electrical nerve stimulation

Sacral nerve stimulation

Spinal cord stimulation

Intravaginal electrical stimulation

Pudendal nerve stimulation

\section{Abstract}

Context: Patients with chronic pelvic pain (CPP) may have pain refractory to conventional pain management strategies.

Neuromodulation could provide relief of pain.

Objective: To evaluate the benefits and harms of neuromodulation for CPP.

Evidence acquisition: A comprehensive search of EMBASE, PUBMED, and SCOPUS was performed for the entire database to January2018. Studies were selected, data were extracted, and quality was assessed by two independent reviewers. A metaanalysis was used to combine randomized controlled trials (RCTs); otherwise, a narrative analysis was used.

Evidence synthesis: After screening 1311 abstracts, 36 studies including eight RCTs were identified, enrolling 1099 patients. Studies covered a broad range in terms of phenotypes of CPP and methods of neuromodulation. A meta-analysis was possible for percutaneous tibial nerve stimulation and transcutaneous electrical nerve stimulation, which showed improvement in pain. Only narrative synthesis was possible for other modalities (sacral nerve stimulation, spinal cord stimulation, intravaginal electrical stimulation, and pudendal nerve stimulation) which appeared to reduce pain in patients with CPP. Treatments generally improved quality of life but with variable reporting of adverse events. Many studies showed high risks of bias and confounding.

Conclusions: While electrical neuromodulation may improve symptoms in CPP, further work is needed with high-quality studies to confirm it.
Patient summary: Neuromodulation may be useful in reducing pain and improving quality of life in patients with chronic pelvic pain, but more research is needed.

\section{Introduction}

Chronic pelvic pain (CPP) is chronic or persistent pain perceived in structures related to the pelvis of men and 1 women; pain must have been continuou s or recurrent for at least 6 mo [1]. The cause of CPP may be unknown (CPP syndrome) or the pain may be due to identifiable disease. The prevalence of CPP has been reported as $5.7 \%$ in women and $2.7 \%$ in men [2]. A recent questionnaire study of adult women in the UK found a prevalence of CPP of $14.8 \%$ [3]. These individuals may suffer significant distress and detriment to their daily living and quality of life (QOL).

While there are various treatment options for individuals with CPP, the efficacy of single-modality treatment is limited [4]. Treatment options include physical treatment (eg, physiotherapy), pharmacological treatment (eg, analgesia, antibiotics, and antidepressants), intravesical treatments, surgical management, or psychological therapy. A combination of pharmacological treatment (such as alpha-blockers, anti-inflammatories, and antibiotics for prostate pain syndrome) may be considered and has been found to confer greater benefit than monotherapy in some conditions [5].

Importantly, there is marked heterogeneity among patients with CPP that complicates evaluation of treatments. A method of phenotyping patients with chronic prostate pain according to presentation as urinary, psycho-social, organspecific, infection, neurological/systemic, and tenderness symptoms has been described [6]. Where management was tailored to the patient's phenotype, there was a significant improvement in QOL and symptoms. When these strategies fail, further therapeutic options can be limited.

Electrical nerve stimulation, in its many forms, has been used to treat pain conditions. The exact mechanism by which neuromodulation achieves pain control is unknown. The gate-control theory of pain proposes that stimulation of larger myelinated afferent nerve fibers can inhibit transmis- 
sion in smaller nociceptive fibers [7]. Newer techniques of neurostimulation suggest that other mechanisms may be involved [8].

Electrical nerve stimulation has also been shown to be effective in the treatment of bladder dysfunction. Patients with refractory overactive bladder and pain were treated with percutaneous tibial nerve stimulation (PTNS), and in addition to an improvement in urinary symptoms, patients reported a significant improvement in pelvic pain $[9,10]$. There are many electrical nerve stimulation techniques and devices. These range from externally applied and noninvasive techniques used in an outpatient setting, such as transcutaneous electrical nerve stimulation (TENS), to implantable and invasive techniques that require sedation (local or general anesthesia), such as sacral nerve stimulation (SNS) or spinal cord stimulation (SCS). As techniques differ so widely, it is important to consider not only their efficacy, but also their safety and adverse effects. The objective of this review was to determine the efficacy and safety of electrical neuromodulation in the treatment of CPP. Primary outcomes are benefit (ie, improvement in pain and QOL) and harm (adverse events following treatment).

\section{Evidence acquisition}

This systematic review was performed according to the Preferred Reporting Items for Systematic Reviews and Meta-Analysis (PRISMA) statement [11]. The protocol for the review is available on PROSPERO (CRD42017054893; https://www.crd.york.ac.uk/PROSPERO/display 78

\subsection{Data sources and searches}

We systematically searched EMBASE, Medline, the Cochrane Central Register of Controlled Trials, and the Health Technology Assessment Database (from 1945 to January 2018). The search strategy is included in the Supplementary 85 material. Titles and abstracts were retained for selection, after search results were combined and deduplicated.

\subsection{Study selection}

There was no restriction on primary study design (ie, to include randomized controlled trials [RCTs], nonrandomized comparative studies, single-arm case series, prospective and retrospective studies, and observational studies). Single-arm case-series were included if there were $>10$ participants and at least one baseline measurement. Case reports, editorial commentaries, and systematic or narrative reviews were excluded. There was no language restriction.

The inclusion criteria required the following: (1) trials with assessment before and after neurostimulation treatment; (2) adult participants with CPP (including all phenotypes of CPP), excluding those undergoing treatment for cancer but not excluding cancer survivors, and excluding pelvic organ prolapse (unless postoperative pain); (3) neuromodulation by any form of electrical neurostimulation; and (4) reporting outcomes included pain (as defined by the trialist).

Two review authors (A.C. and S.G.) independently screened titles and abstracts of identified records to identify Potentially eligible trials, and then obtained full papers to determine the final set of studies. Where there was discrepancy between reviewers, a third reviewer was consulted (M.S.) and consensus was achieved.

\subsection{Data extraction and risk of bias}

Full text of potentially eligible studies was reviewed and data were extracted. Variables extracted were (wherever available) the following: year of publication, number and sex of participants, age of participants, type of pain syndrome, mean duration of symptoms, type of intervention, and specifics of stimulation (including protocol, frequency, pulse width, and amplitude). Outcomes were pain and adverse events (primary outcomes) and QOL (secondary outcomes). Data were extracted by two reviewers, and discrepancies were resolved as before. Where information was missing, authors were contacted.

For RCTs, the Cochrane Risk of Bias Assessment tool was used, including assessment of sequence generation (selection bias), allocation concealment (selection bias), blinding of participants, personnel and outcome assessors (performance bias), incomplete outcome data (attrition bias), and selective outcome reporting (reporting bias) [12]. For non-RCT studies, the Cochrane tool was used, and in addition, an a priori list of confounders was identified with clinical content experts (members of the European Association of Urology [EAU] Chronic Pelvic Pain Guidelines Panel). This enabled consideration of each confounder and determination of whether it was controlled for. These potential confounders were sex of patients, phenotype of CPP, presence of bowel or bladder dysfunction, distress or catastrophizing, and type of neurostimulation (including parameters and duration of treatment).

\subsection{Data synthesis}

For each of the included RCT studies using pain score (out of 10), we calculated the effect size (ES) and corresponding $95 \%$ confidence intervals (CIs). Since data were sparse, the meta-analysis addressed broad questions across study design. We calculated the overall standard mean difference between treatment and control groups using a random-effect model. Forest plots were generated in order to provide a visual representation of the results and to illustrate the direction and magnitude of effects.

No pooling was planned for non-RCTs due to different study designs and the expected clinical and methodological heterogeneity of included studies, but forest plots were generated to provide a visual representation of results to show the direction and magnitude of effects before and after treatment in studies reporting primary outcome of pain as a pain score.

Analyses were performed using the "metan" command of the Stata statistics software package (Stata 14.0 and 9.0 statistics software package, StataCorp 2009 Stata Statistical, SoftwardeRelease 14; StataCorp LP, College Station, TX, USA). Risk of bias summary and graph (Supplementary Fig. 1 and 2) were generated using Cochrane RevMan software v5.3 (Informatics and Knowledge Management Department, Cochrane, London, UK).

\section{Evidence synthesis}

3.1. Search results

The PRISMA diagram illustrates the literature search and results (Fig. 1). The final 36 studies consisted of eight RCTs, four randomized noncontrolled trials, one crossover trial, 18 prospective cohort studies, and five retrospective case series studies.

\subsection{Study and patient characteristics}

Table 1 describes the characteristics of included studies. Tables 2-4 describe outcomes of RCTs and non-RCTs.

3.3. Benefits and harms of electrical neuromodulation techniques Fig. 2A shows the meta-analysis of the difference in pain scores between treatment groups and control in RCTs. An overall ES of -2.41 (95\% CI $-2.87,-1.95)$ was found, representing statistically significant benefit of treatment over control and translating to an improvement in pain score of 2.4/10, a clinically meaningful amount. For non-RCTs, Fig. 2B and $\mathrm{C}$ show forest plots of change in pain scores before and after the procedure by condition and treatment, respectively.

3.3.1. Benefits and harms of SNS 
A total of 10 studies evaluating the efficacy of SNS were identified [13-22], comprising six prospective cohort studies $[13-15,17,19,22]$ and four retrospective case series $[16,18,20,21]$. No RCTs were identified. Follow-up ranged from 4 to $239 \mathrm{mo}$. Pain conditions as defined by authors included CPP, bladder pain syndrome (BPS), and interstitial cystitis (IC; Table 1). Where reported, a mean of $69 \%$ of patients undergoing test stimulation proceeded to formal implantation (range 52-91\%).

All studies reported a decrease in pain score following SNS. In some studies, the primary outcome was reported as a pain score (out of 10), which is illustrated in Fig. 2C; this was statistically significant in five studies [14,16-18,22] although not significant in one [13]. A decrease in score following treatment ranged from 3.1 to $6.5 / 10$ [13-17], with a mean reduction of $4.4 / 10$. One study reported a marked improvement in pelvic pain in $71 \%$ of participants [19]; another long-term study found that $64 \%$ of patients reported no pelvic pain at the last clinic visit with average follow-up of approximately $5 \mathrm{yr}$ [20].

QOL parameters were measured in three studies $[17,21,22]$. There was some statistically significant improvement in QOL, as measured by the Short Form (36) Health Survey (SF-36) questionnaire in two studies, including general health, bodily pain and social functioning, and physical domains, social functioning, and mental health $[17,21]$, but another study reported no statistically significant improvement in QOL following SNS.

Safety of SNS was reported in all 10 studies. No adverse events were described in two studies $[14,17]$. There was a large variation in adverse events and in details reported. The reported rates of adverse events ranged from $0 \%$ to $50 \%$. Events not requiring reoperation included pain, failure of function of device, wound infection, and seroma. Where reported, reoperation rate ranged from $11 \%$ to $50 \%$ [18-20,22]. Indication for reoperation included lead migration, malfunction, systemic infection, intrathecal implantation, erosion, and loss of efficacy (Table 1).

\subsubsection{Benefits and harms of PTNS}

Six studies, three RCTs, and three non-RCTs evaluated the

efficacy of PTNS in CPP [23-28]. Follow-up ranged from 12 to 24 wk. Pain conditions were CPP, IC, and BPS.

All three RCTs demonstrated a statistically significant reduction in pain scores, with the mean reduction of score ranging from 3.3 to $5.3 / 10$; no significant reduction was seen in control groups (no treatment or sham PTNS) [23-25]. In two of three non-RCTs, there was a significant reduction in pain score following treatment for CPP and IC $[26,28]$, but the third study demonstrated no significant improvement in pain for BPS (Table 3) [27]. One RCT evaluating long-term effect of PTNS found that the improvement in pain score in PTNS group was maintained at 6-mo follow-up [24]. All three RCTs examined QOL following PTNS for CPP. All demonstrated a statistically significant improvement in QOL as measured by the SF36 $[23,24]$ and National Institute of Health Chronic Prostatitis Index (NIH-CPSI)/QOL domain [25]. At 6 mo after the procedure, a continued significant improvement in the social functioning score was observed [24]. QOL was measured in two non-RCTs, demonstrating a significant improvement in QOL measured by SF-36 in one study [28], but no improvement in QOL as measured using the International Prostate Symptom Score QOL domain in another [26].

Adverse events were rare following PTNS and reported in three of six studies $[23,24,26]$. Temporary slight pain at the surgical site was described in all studies where adverse events were reported and hematoma in one patient [23].
Twelve studies, including four RCTs, evaluated the efficacy of TENS for CPP [29-40]. One RCT combined TENS and thermotherapy [31]. Follow-up of RCTs ranged from 4 to $12 \mathrm{wk}$ (not recorded in one study). Follow-up of non-RCTs ranged from immediately following treatment to 40 wk. Pain conditions were dysmenorrhea, endometriosisrelated pain, $\mathrm{CPP}$, provoked vestibulodynia (PV), and prostate pain syndrome.

Three RCTs found a statistically significant reduction in pain following TENS therapy (mean reduction of 1.9 and $4 / 10$ ) or NIH-CPSI pain domain, compared with either sham or placebo $[29,30,32]$. When TENS was combined with thermotherapy and compared with sham, a significant reduction of 1.8/10 was reported [31].

Regarding non-RCTs, a statistically significant reduction in pain score was reported in three studies [38-40]. Two studies of TENS for dysmenorrhea reported a moderate or marked improvement in pain in $91.2 \%$ and $87.3 \%$ of patients, although $9.8 \%$ and $12.7 \%$, respectively, reported no or mild improvement $[33,34]$. One study compared two methods of TENS (self-applied and acupuncture-like TENS) in women with endometriosis and demonstrated a statistically significant improvement in pain in both treatment modalities with a mean overall improvement of $3.5 / 10[35]$.

QOL outcomes were measured in five studies [29-31,35,36]. Two RCTs comparing TENS and TENS plus thermotherapy with sham for dysmenorrhea found no significant improvement in QOL as measured by the WHO QOL-BREF questionnaire [29,31]. Another RCT evaluated QOL following TENS for dysmenorrhea versus sham, and demonstrated a statistically significant improvement in trialist-defined QOL outcomes including capacity to get out of bed, food/drink intake, and quality of sleep, but no significant improvement in daily activities [30]. A randomized study comparing modes of TENS (self-applied and acupuncture-like TENS) reported whole-group results for QOL and found a statistically significant improvement in the endometriosis QOL score [35]. Another study reported a statistically significant improvement in QOL following TENS in men with CPP. Trialist-defined improvement in QOL by patient satisfaction was described as follows: prior to treatment, all patients felt dissatisfied, unhappy, or terrible, and after treatment, $48 \%$ were mostly satisfied, pleased, or delighted.

Two studies reported longer-term outcomes of patients after TENS treatment had been withdrawn [36]. One study reported a successful outcome in $45 \%$ of men treated with a 12-wk course of TENS for CPP; at a mean follow-up of 43.6 mo, the effect was retained in $72 \%$ of these men [36]. Another study of women with PV reported a statistically significant improvement in pain at a mean duration of use of $6.2 \mathrm{mo}$. At a mean follow-up of $10.1 \mathrm{mo}$ (after 12-16 wk of treatment), it was found that this effect was maintained [35].

\subsubsection{Efficacy of other methods of neuromodulation}

Two studies evaluated intravaginal electrical stimulation (IES) in women with CPP $[41,42]$. One randomized crossover trial compared active with placebo IES. While pain was measured, results were reported as a proportion of women with a pain score of $3 / 10$ after treatments. At baseline, $27.3 \%$ of those receiving placebo followed by active treatment, and $20 \%$ of those receiving active treatment followed by placebo reported pain scores of $<3.10$. Following treatment, there was a statistically significant reduction in pain for both placebo and treatment groups, but the effect was more noticeable for active treatment [42]. Those undergoing active followed by placebo treatment ( $86.7 \%$ and $78.6 \%$, respectively) reported a pain score of $<3$. The group undergoing placebo followed by active treatment $(54.5 \%$ and $90.9 \%$, respectively) reported a pain score of $<3$ 
There were no adverse events, and QOL outcomes were not assessed. A prospective series of women with CPP by the same authors reported a significant reduction in pain, with a mean reduction of 6.2/10 continuing 7 mo after treatment [41]. QOL outcomes were not assessed and adverse events were not reported.

Pudendal nerve stimulation (PNS) was examined in two studies. In a prospective pilot study evaluating PNS for CPP, there was a statistically significant reduction in the pain score measured at 4-wk follow-up [43]. No QOL measures were assessed, nor were any adverse events reported. Another prospective pilot study of patients with pudendal neuralgia reported subjective response rates of patients at $2 \mathrm{wk}$, and demonstrated a $36 \%$ rate of complete or almost complete pain relief, 52\% rate of significant/remarkable pain relief, and $16 \%$ rate of slight/small pain relief compared with nerve block. Of the patients, $26 \%$ underwent explantation of the device. Adverse events and QOL outcomes were not measured [44]. One prospective cohort study evaluated SCS for pudendal neuralgia. In those patients who responded to test stimulation, there was a significant reduction in pain with a mean reduction in of 2.9/10 [45]. QOL outcomes were not measured, and no adverse events reported.

Transcutaneous interferential electrical stimulation (TIES) was evaluated by one RCT, comparing it with sham TIES in patients with irritable bowel syndrome (IBS), demonstrating a significant reduction in pain for both the treatment and the placebo group. The decrease in pain score continued, and was statistically significant in both the treatment and the placebo group at follow-up in the $1 \mathrm{st}$ month of treatment. In the treatment group, there was a statistically significant improvement only in QOL, as measured by total IBS-QOL score. No group comparisons and adverse events were reported [46]. Another study randomized women with dysmenorrhea to either TENS or TIES [39]. There was a significant reduction in pain for both groups with no adverse events; QOL outcomes were not evaluated.

Electrical acupuncture (high- and low-frequency modes) was compared with manual acupuncture in a trial of women with dysmenorrhea [47]. Primary outcome was pain at 12 mo, but only $38 \%$ of women completed treatment and 12 -mo data were available for $28 \%$ of women. While there was a significant improvement in pain scores in all groups, there was no difference in the mode of stimulation or frequency. At 1-mo follow-up, there was a significant improvement in QOL, as measured by the SF-36 questionnaire in the total physical component for electrical compared with manual acupuncture. Adverse events were reported in $7.4 \%$ including hematoma, soreness, and fatigue.

Another study compared TENS with different methods of acupuncture, including manual, low-, and high-frequency electrical acupuncture, in women with dysmenorrhea and found a statistically significant improvement in pain score in all modes of acupuncture treatment [38]. QOL and adverse events were not reported.

One retrospective study compared electrical stimulation plus biofeedback with electromagnetic stimulation in men with CPP. Both treatment modalities reported a statistically significant improvement in visual analog scale, with a mean pain reduction of 2.5/10 in the ES group. A statistically significant improvement in QOL as measured by the QOL domain of the NIH-CPSI was demonstrated. Adverse events were not reported [48].

\subsection{Risk of bias and confounding}

There was a notable risk of bias in both RCTs and nonRCTs, as shown by Supplementary Figs. 1 and 2. In RCTs, this was most commonly blinding, selection, and performance bias. In non-RCTs, selective outcome reporting and attrition bias was found in the majority of studies, which could lead to inflation of treatment benefits. Across the entire study set, power calculations were performed in nine studies in total, of which only six were adequately powered.

\subsection{Discussion}

\subsubsection{Principal findings}

This study systematically reviewed the efficacy and safety of neuromodulation in patients with CPP. There was a wide range of pain conditions and treatment modalities, but overall neuromodulation produced a reduction in pain and, in the trials that assessed it, in QOL scores, with no major safety problems. The risk of bias and confounding was high, particularly for the nonrandomized studies, and hence results should be interpreted with caution.

\subsubsection{Findings in the context of existing evidence}

Neuromodulation may be an effective treatment in patients with idiopathic overactive bladder. A recent systematic review of SNS and PTNS found that while both modalities gave promising results in terms of improvements in overactive bladder symptoms, studies were limited by poor quality [49]. Therefore, in patients with voiding dysfunction and pain, refractory to standard treatment, neuromodulation techniques could offer treatment improving both pain and urinary symptoms.

\subsubsection{Implications for research}

The highest quality of evidence for systematic reviews is from appropriately powered RCTs. Ideally, further largescale RCTs are needed in all neuromodulation modalities. Long-term outcome data are scarce; therefore, future research should include evaluation of lasting effects of treatment. A precise definition of participants and CPP subgroup or phenotype should be used. Primary endpoints should be standardized and established QOL measures should be applied. QOL outcomes should be measured in addition to pain as significant QOL improvements may be noted without discernible change in pain. Adverse events should be reported, including the time when they occur in a standardized form. All parameters for stimulation need to be 432 clearly stated and recorded (eg, pulse width, frequency of stimulation and amplitude, perceived intensity, and technique of establishing the end point for electrode insertion).

\subsubsection{Implications for practice}

CPP can prove difficult to treat satisfactorily, and a holistic approach tailored to an individual patient is recommended using clinical experience [1]. The neurostimulation techniques described in this review are varied and differ in invasiveness and side-effect profiles, so each patient should be provided with sufficient information about the alternatives proposed to make an informed decision on which treatment to consider. TENS has been shown to be an effective treatment for women with CPP secondary to dysmenorrhea and is free from adverse events, with the advantage that it can be self-applied and cost effective. Similarly, PTNS has been shown to be effective in a variety of pain conditions with minimal complications; however, it is time consuming with current routinely available approaches. More invasive techniques, such as SNS, require a trial period of stimulation (after which a number of patients will not continue). While patients may achieve symptomatic relief, this should be weighed up against a higher complication rate. Similarly with nonneuromodulation techniques, the aim is not only pain relief but also improved function, although this may not be achievable [50]. It is important to bear in mind that QOL may be affected directly by both functional improvement and pain reduction.

Neuromodulation techniques may not be available to all 
patients due to geographical or financial constraints. As robust evidence is not currently available, patients should ideally undergo treatment at tertiary centers under expert practitioners and preferably as part of a trial.

3.5.5. Limitations of this study

Studies identified in the review were largely of poor quality with a significant risk of bias. Only eight RCTs were identified, and there is a risk of publication bias, whereby studies with negative findings are not published but could affect the overall effects of treatment estimated in this paper. Several studies were very likely underpowered, and where power calculations were performed, only a third were adequately powered. Patients with CPP are a heterogeneous group with multiple definitions, and there were not sufficient data to estimate the therapeutic benefit for subgroups included in the review, particularly given the large variety of treatment protocols. Further, follow-up is insufficient to show treatment gains over a realistic time frame for a chronic problem, limiting clinical generalizability.

While the primary outcome of pain reduction was described in all studies, the method of reporting this outcome differed. QOL outcomes were reported in less than half the studies, and well-established QOL scales were not always used. Adverse events were reported in just over half of studies examined, but should be recorded in all trials.

The strength of this review is that it adhered to the published protocol and followed search criteria devised by members of the EAU CPP Guideline Panel. Practitioners were involved at all stages to ensure that results were clinically useful. A sufficient number of RCTs were identified to perform an overall meta-analysis. The weaknesses of this review were possible publication bias, and the lack of data from original studies to allow more specific conclusions about subgroups or methods of neuromodulation. No response was received when authors were contacted for further information.

\section{Conclusions}

Neuromodulation may provide an effective treatment option in patients with CPP refractory to standard treatment, reducing pain and improving QOL with an acceptable rate of complications, but study quality is insufficient for a more certain conclusion. Quality of studies was generally poor, and therefore larger-scale, well-designed, and powered RCTs with long-term outcomes are needed.

Author contributions: Angela M. Cottrell had full access to all the data in the study and takes responsibility for the integrity of the data and the accuracy of the data analysis.

Study concept and design: Cottrell, Schneider, Goonewardene, Baranowski, Engeler, Borovicka, Dinis-Oliveira, Elneil, Hughes, Messelink, de C Williams.

Acquisition of data: Cottrell, Schneider, Yuan.

Analysis and interpretation of data: Cottrell, Schneider, de C Williams.

Drafting of the manuscript: Cottrell, de C Williams.

Critical revision of the manuscript for important intellectual content:

Goonewardene, Baranowski, Engeler, Borovicka, Dinis-Oliveira, Elneil, Hughes, Messelink, de C Williams.

Statistical analysis: Schneider.

Obtaining funding: None

Administrative, technical, or material support: Cottrell, Schneider, Goonewardene.

Supervision: de C Williams, Baranowski, Engeler.

Other: None.

Financial disclosures: Angela M. Cottrell certifies that all conflicts of interest, including specific financial interests and relationships and affiliations relevant to the subject matter or materials discussed in the manuscript (eg, employment/affiliation, grants or funding, consultancies, honoraria, stock ownership or options, expert testimony, royalties, or patents filed, received, or pending), are the following: Dr. A.C. de C Williams is the author of a Cochrane Systematic Review on pelvic pain in women and nonsurgical interventions, and is in the editorial board of a number of pain journals. Dr. A.P. Baranowski is the owner of a private medical practice. Dr. J. Hughes holds the following positions: Faculty of
Pain Medicine of the Royal College of Anaesthetists—elected board member from 2013 to 2019, current Vice Dean 2016-date; NHS England: Clinical Reference Group for Specialised Pain Services-member for the North East (2012-2014), chair 2014-2019; IASP-IASP Educational Working Group (2009-2016). He was also one of the editors of the book Abdominal and Pelvic Pain: from Definition to Best Practice (published 2014). Dr. A.M. Cottrell attended a course sponsored by Boston Scientific in November 2018. M.P. Schneider, S. Goonewardene, D.E. Engeler, J. Borovicka, P. Dinis-Oliveira, S. Elneil, and B.E.J. Messelink have nothing to declare.

Funding/Support 539 and role of the sponsor: None.

Appendix

A. Supplementary data

Supplementary material related to this article can be found, in the online version, at doi:https://doi.org/10. 1016/j.euf.2019.09.011.

References

[1] Engeler DE BA, Borovicka J, Cottrell A, et al. EAU guidelines chronic pelvic pain. https://uroweb.org/guideline/chronic-pelvicpain/2017

[2] Marszalek M, Wehrberger C, Temml C, Ponholzer A, Berger I, Madersbacher S. Chronic pelvic pain and lower urinary tract symptoms in both sexes: analysis of 2749 participants of an urban health screening project. Eur Urol 2009;55:499-507.

[3] Ayorinde AA, Bhattacharya S, Druce KL, Jones GT, Macfarlane GJ. Chronic pelvic pain in women of reproductive and post-reproductive age: a population-based study. Eur J Pain 2017;21:445-55. [4] Cheong YC, Smotra G, Williams AC. Non-surgical interventions for The management of chronic pelvic pain. Cochrane Database Syst Rev 2014;3:3.

[5] Thakkinstian A, Attia J, Anothaisintawee T, Nickel JC. Alpha-blockers, antibiotics and anti-inflammatories have a role in the management of chronic prostatitis/chronic pelvic pain syndrome. BJU Int 2012;110:1014-22

[6] Shoskes DA, Nickel JC, Kattan MW. Phenotypically directed multimodal therapy for chronic prostatitis/chronic pelvic pain syndrome: a prospective study using UPOINT. Urology 2010;75: 1249-53.

[7] Melzack R, Wall PD. Pain mechanisms: a new theory. Science 1965;150:971-9.

[8] Kapural L, Yu C, Doust MW, et al. Comparison of 10-kHz highfrequency and traditional low-frequency spinal cord stimulation for the treatment of chronic back and leg pain: 24-month results from a multicenter, randomized, controlled pivotal trial. Neurosurgery 2016;79:667-77

[9] Govier FE, Litwiller S, Nitti V, Kreder Jr KJ, Rosenblatt P. Percutaneous afferent neuromodulation for the refractory overactive bladder: results of a multicenter study. J Urol 2001;165:1193-8.

[10] Congregado Ruiz B, Pena Outeirino XM, Campoy Martinez P, Leon Duenas E, Leal Lopez A. Peripheral afferent nerve stimulation for treatment of lower urinary tract irritative symptoms. Eur Urol 2004;45:65-9.

[11] Moher D, Liberati A, Tetzlaff J, Altman DG, Group P. Preferred reporting items for systematic reviews and meta-analyses: the PRISMA statement. Ann Intern Med 2009;151:264-9, W64. [12] Viswanathan M, Ansari MT, Berkman ND, et al. Assessing the risk of bias of individual studies in systematic reviews of health care interventions. Methods guide for effectiveness and comparative effectiveness reviews AHRQ methods for effective health care. Rockville, MD: AHRQ; 2008

[13] Aboseif S, Tamaddon K, Chalfin S, Freedman S, Kaptein J. Sacral neuromodulation as an effective treatment for refractory pelvic floor dysfunction. Urology 2002;60:52-6.

[14] Comiter CV. Sacral neuromodulation for the symptomatic treatment of refractory interstitial cystitis: a prospective study. J Urol 2003;169:1369-73.

[15] Feler CA, Whitworth LA, Brookoff D, Powell R. Recent advances: sacral nerve root stimulation using a retrograde method of lead insertion for the treatment of pelvic pain due to interstitial cystitis. Neuromodulation 1999:2:211-6.

[16] Ghazwani YQ, Elkelini MS, Hassouna MM. Efficacy of sacral neuromodulation in treatment of bladder pain syndrome: long-term follow-up. Neurourol Urodyn 2011;30:1271-5.

[17] Maher CF, Carey MP, Dwyer PL, Schluter PL. Percutaneous sacral nerve root neuromodulation for intractable interstitial cystitis.

J Urol 2001;165:884-6.

[18] Marinkovic SP, Gillen LM, Marinkovic CM. Minimum 6-year outcomes for interstitial cystitis treated with sacral neuromodulation. Int Urogynecol J Pelvic Floor Dysfunct 2011;22:407-12.

[19] Peters KM, Carey JM, Konstandt DB. Sacral neuromodulation for 
the treatment of refractory interstitial cystitis: outcomes based on technique. Int Urogynecol J Pelvic Floor Dysfunct 2003;14 223-8.

[20] Powell CR, Kreder KJ. Long-term outcomes of urgency-frequency syndrome due to painful bladder syndrome treated with sacral neuromodulation and analysis of failures. J Urol 2010;183:173-6.

[21] Siegel S, Paszkiewicz E, Kirkpatrick C, Hinkel B, Oleson K. Sacral nerve stimulation in patients with chronic intractable pelvic pain. $\mathrm{J}$ Urol 2001;166:1742-5.

[22] Zabihi N, Mourtzinos A, Maher MG, Raz S, Rodriguez LV. Shortterm results of bilateral S2-S4 sacral neuromodulation for the treatment of refractory interstitial cystitis, painful bladder syndrome, and chronic pelvic pain. Int Urogynecol J Pelvic Floor Dysfunct 2008;19:553-7.

[23] Gokyildiz S, Kizilkaya Beji N, Yalcin O, Istek A. Effects of percutaneous tibial nerve stimulation therapy on chronic pelvic pain. Gynecol Obstet Invest 2012;73:99-105.

[24] Istek A, Gungor Ugurlucan F, Yasa C, Gokyildiz S, Yalcin O. Randomized trial of long-term effects of percutaneous tibial nerve stimulation on chronic pelvic pain. Arch Gynecol Obstet 2014;290:2918.

[25] Kabay S, Kabay SC, Yucel M, Ozden H. Efficiency of posterior tibial nerve stimulation in category IIIB chronic prostatitis/ chronic pelvic pain: a sham-controlled comparative study. Urol Int 2009;83:33-8.

[26] Kim SW, Paick JS, Ku JH. Percutaneous posterior tibial nerve stimuation in patients with chronic pelvic pain: a preliminary study. Urol Int 2007;78:58-62.

[27] Ragab MM, Tawfik AM, Abo El-Enen M, et al. Evaluation of percutaneous tibial nerve stimulation for treatment of refractory painful bladder syndrome. Urology 2015;86:707-11.

[28] Van Balken MR, Vandoninck V, Messelink EJ, et al. Percutaneous tibial nerve stimulation as neuromodulative treatment of chronic pelvic pain. Eur Urol 2003;43:158-63.

[29] Bai H-Y, Bai H-Y, Yang Z-Q. Effect of transcutaneous electrical nerve stimulation therapy for the treatment of primary dysmenorrheal. Medicine 2017;96:e7959.

[30] Lauretti GR, Oliveira R, Parada F, Mattos AL. The new portable transcutaneous electrical nerve stimulation device was efficacious in the control of primary dysmenorrhea cramp pain. Neuromodulation 2015;18:522-7.

[31] Lee B, Hong SH, Kim K, et al. Efficacy of the device combining high-frequency transcutaneous electrical nerve stimulation and thermotherapy for relieving primary dysmenorrhea: a randomized, single-blind, placebo-controlled trial. Eur J Obstet Gynecol Reprod Biol 2015;194:58-63.

[32] Sikiru L, Shmaila H, Muhammed SA. Transcutaneous electrical nerve stimulation (TENS) in the symptomatic management of chronic prostatitis/chronic pelvic pain syndrome: a placebo-control randomized trial. Int Braz J Urol 2008:34:708-14.

[33] Kaplan B, Peled Y, Pardo J, et al. Transcutaneous electrical nerve stimulation (TENS) as a relief for dysmenorrhea. Clin Exp Obstet Gynecol 1994;21:87-90.

[34] Kaplan B, Rabinerson D, Lurie S, Peled Y, Royburt M, Neri A. Clinical evaluation of a new model of a transcutaneous electrical nerve stimulation device for the management of primary dysmenorrhea. Gynecol Obstet Invest 1997;44:255-9.

[35] Mira TAA, Giraldo PC, Yela DA, Benetti-Pinto CL. Effectiveness of complementary pain treatment for women with deep endometriosis through transcutaneous electrical nerve stimulation (TENS): randomized controlled trial. Eur J Obstet Gynecol Reprod Biol 2015;194:1-6.

[36] Schneider MP, Tellenbach M, Mordasini L, Thalmann GN, Kessler TM. Refractory chronic pelvic pain syndrome in men: Can transcutaneous electrical nerve stimulation help? BJU Int 2013;112: E159-63.

[37] Schiotz HA, Jettestad M, Al-Heeti D. Treatment of dysmenorrhoea with a new TENS device (OVA). J Obstet Gynaecol 2007;27:726-8

[38] Thomas M, Lundeberg T, Bjork G, Lundstrom-Lindstedt V. Pain and discomfort in primary dysmenorrhea is reduced by preemptive acupuncture or low frequency TENS. Eur J Phys Med Rehabil 1995;5:71-6.

[39] Tugay N, Akbayrak T, Demirturk F, et al. Effectiveness of transcutaneous electrical nerve stimulation and interferential current in primary dysmenorrhea. Pain Med 2007:8:295-300. [40] Vallinga MS, Spoelstra SK, Hemel IL, van de Wiel HB, Weijmar Schultz WC. Transcutaneous electrical nerve stimulation as an additional treatment for women suffering from therapy-resistant provoked vestibulodynia: a feasibility study. J Sex Med 2015;12: 228-37. [41] De Oliveira Bernardes N, Bahamondes L. Intravaginal electrical stimulation for the treatment of chronic pelvic pain. J Reprod Med Obstet Gynecol 2005;50:267-72.

[42] De Oliveira Bernardes N, Marques A, Ganunny C, Bahamondes L. Use of intravaginal electrical stimulation for the treatment of chronic pelvic pain: a randomized, double-blind, crossover clinical trial. J
Reprod Med Obstet Gynecol 2010;55:19-24.

[43] Heinze K, Hoermann R, Fritsch H, Dermietzel R, van Ophoven A. Comparative pilot study of implantation techniques for pudendal neuromodulation: technical and clinical outcome in first 20 patients with chronic pelvic pain. World J Urol 2015;33:289-94.

[44] Peters KM, Killinger KA, Jaeger C, Chen C. Pilot study exploring chronic pudendal neuromodulation as a treatment option for pain associated with pudendal neuralgia. Low Urin Tract Symptoms 2015;7:138-42.

[45] Buffenoir K, Rioult B, Hamel O, Labat JJ, Riant T, Robert R. Spinal cord stimulation of the conus medullaris for refractory pudendal neuralgia: a prospective study of 27 consecutive cases. Neurourol Urodyn 2015;34:177-82.

[46] Coban S, Akbal E, Koklu S, et al. Clinical trial: transcutaneous interferential electrical stimulation in individuals with irritable bowel syndrome- a prospective double-blind randomized study. Digestion 2012;86:86-93

[47] Armour M, Dahlen HG, Zhu X, Farquhar C, Smith CA. The role of treatment timing and mode of stimulation in the treatment of primary dysmenorrhea with acupuncture: an exploratory randomised controlled trial. PLoS One 2017;12:e0180177.

[48] Yang MH, Huang YH, Lai YF, Zeng SW, Chen SL. Comparing electromagnetic stimulation with electrostimulation plus biofeedback in treating male refractory chronic pelvic pain syndrome. Urol Sci 2017;28:156-61

[49] Tutolo M, Ammirati E, Heesakkers J, et al. Efficacy and safety of sacral and percutaneous tibial neuromodulation in non-neurogenic lower urinary tract dysfunction and chronic pelvic pain: a systematic review of the literature. Eur Urol 2018. http://dx.doi.org/10. 1016/j.eururo.2017.11.002, In press.

[50] Noble M, Treadwell JR, Tregear SJ, et al. Long-term opioid manageMent for chronic noncancer pain. Cochrane Database Syst Rey 2010;1:CD006605. 


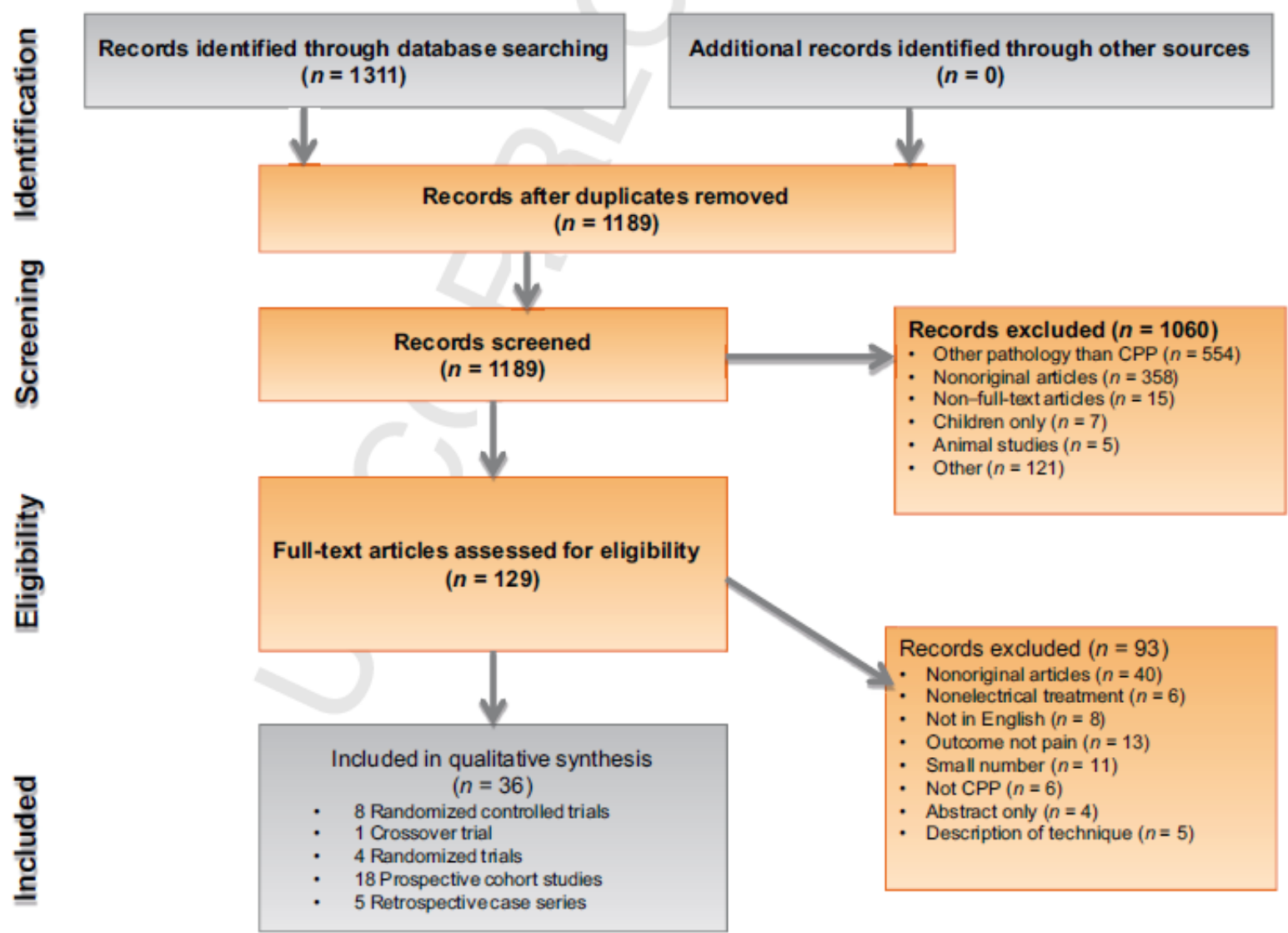

Fig. 1 - PRISMA flow diagram. CPP= chronic pelvic pain; PRISMA= Preferred Reporting Items for Systematic Reviews and Meta-Analysis. 


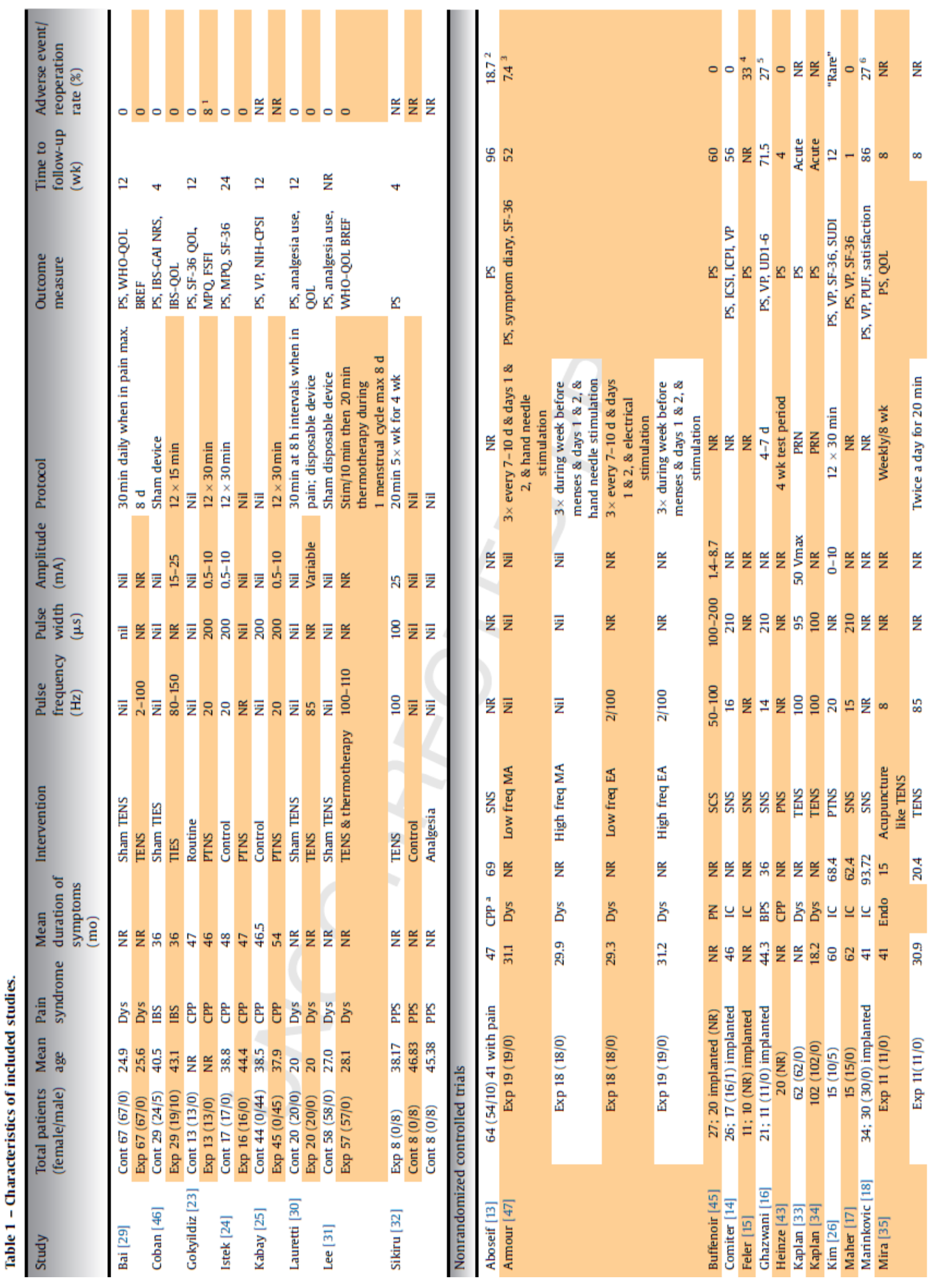




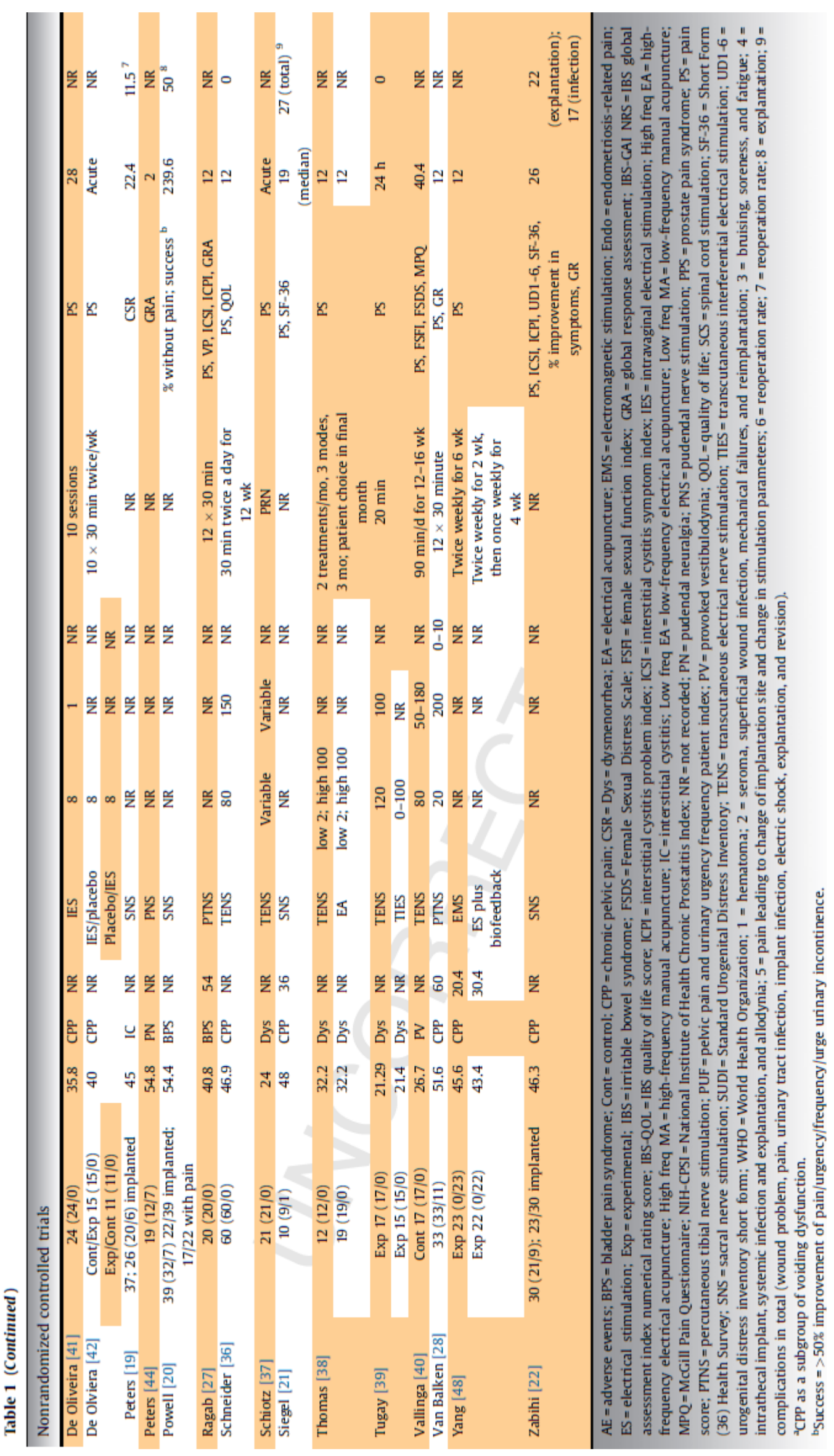


Table 2 - Treatment outcomes of randomized controlled studies.

\begin{tabular}{|c|c|c|c|c|c|c|c|c|c|c|c|c|}
\hline \multicolumn{3}{|l|}{ Author } & \multicolumn{10}{|c|}{ Pain scores } \\
\hline & $\begin{array}{c}\text { Total } \\
\text { patients }\end{array}$ & Group & $\begin{array}{l}\text { NRS } \\
\text { before }\end{array}$ & $\mathrm{SD}$ & $\begin{array}{l}\text { NRS } \\
\text { after }\end{array}$ & SD & $p$ value & $\begin{array}{c}\text { NIH-CPI } \\
\text { pain index } \\
\text { before }\end{array}$ & $\mathrm{SD}$ & $\begin{array}{l}\text { NIH-CPI } \\
\text { pain index } \\
\text { after }\end{array}$ & $\mathrm{SD}$ & $p$ value \\
\hline \multirow[t]{2}{*}{ Bai [29] } & 67 & Cont & $7.2 / 10$ & 1.4 & $6.7 / 10$ & NR & $<0.01$ & NR & NR & NR & NR & NR \\
\hline & 67 & Exp & $7.3 / 10$ & 1.4 & $5.4 / 10$ & NR & $<0.01$ & NR & NR & NR & NR & NR \\
\hline \multirow[t]{2}{*}{ Coban [46] } & 29 & Cont & $66.6 / 100$ & 23.4 & $28.1 / 100$ & 26.5 & $<0.001$ & NR & NR & NR & NR & NR \\
\hline & 29 & Exp & $56 / 100$ & 20 & $21.3 / 100$ & 20.9 & $<0.001$ & NR & NR & NR & NR & NR \\
\hline \multirow[t]{2}{*}{ Gokyildiz [23] } & 13 & Cont & $7.95 / 10$ & 1.03 & $7.87 / 10$ & 0.88 & NR & NR & NR & NR & NR & NR \\
\hline & 13 & Exp & $8.08 / 10$ & 1.72 & $2.62 / 10$ & 2.7 & NR & NR & NR & NR & NR & NR \\
\hline \multirow[t]{2}{*}{ Istek [24] } & 17 & Cont & $6.5 / 10$ & 1.1 & $6 / 10$ & 1.5 & 0.213 & NR & NR & NR & NR & NR \\
\hline & 16 & Exp & $8.4 / 10$ & 1.1 & $3.8 / 10$ & 3.5 & 0.001 & NR & NR & NR & NR & NR \\
\hline \multirow[t]{2}{*}{ Kabay [25] } & 44 & Cont & $7.4 / 10$ & 0.9 & $7.2 / 10$ & 0.4 & $>0.05$ & NR & NR & NR & NR & NR \\
\hline & 45 & Exp & $7.6 / 10$ & 0.8 & $4.3 / 10$ & 0.6 & $<0.001$ & NR & NR & NR & NR & NR \\
\hline \multirow[t]{2}{*}{ Lauretti [30] } & 20 & Cont & $8 / 10$ & NR & $7 / 10$ & NR & NR & NR & NR & NR & NR & NR \\
\hline & 20 & Exp & $8 / 10$ & NR & $2 / 10$ & NR & $<0.001$ & NR & NR & NR & NR & NR \\
\hline \multirow[t]{2}{*}{ Lee [31] } & 58 & Cont & $5.98 / 10$ & 1.36 & $5.64 / 10$ & 1.58 & NR & NR & NR & NR & NR & NR \\
\hline & 57 & Exp & $6.01 / 10$ & 1.03 & $4.23 / 10$ & 1.5 & $<0.001$ & NR & NR & NR & NR & NR \\
\hline \multirow[t]{3}{*}{ Sikiru [32] } & 8 & Cont & NR & NR & NR & NR & NR & 20.25 & 3.73 & 15.88 & 1.55 & NS \\
\hline & 8 & Exp & NR & NR & NR & NR & NR & 16.38 & 2.88 & 9 & 0.93 & $<0.05$ \\
\hline & 8 & Analgesic & NR & NR & NR & NR & NR & 17.13 & 4.91 & 13.38 & 1.5 & NS \\
\hline
\end{tabular}

Exp = experimental; NIH-CPI = National Institute of Heal th Chronic Prostatitis Symptom Index; NR = not recorded; NRS = Numeric Rating Scale; Cont =control; $\mathrm{NS}=$ not significant; $\mathrm{SD}=$ standard deviation.

Table 3 - Pain outcomes of non-RCT.

\begin{tabular}{|c|c|c|c|c|}
\hline Author & No. of patients/therapy & NRS before & NRS after & $p$ value \\
\hline Aboseif [13] & $41 / \mathrm{SNS}$ & $5.8 / 10$ & $3.7 / 10$ & $>0.05$ \\
\hline \multirow[t]{4}{*}{ Armour [47] } & 19/low freq MA & $5.5 / 10$ & $4 / 10$ & $<0.001$ \\
\hline & 18/high freq MA & $4.4 / 10$ & $2.9 / 10$ & $<0.001$ \\
\hline & 18/low freq EA & $5.5 / 10$ & $4.2 / 10$ & $<0.001$ \\
\hline & 19/high freq EA & $5.7 / 10$ & $4.2 / 10$ & $<0.001$ \\
\hline Buffenoir [45] & $20 / S C S$ & $55.0 / 100$ & $26.2 / 100$ & $<0.001$ \\
\hline Comiter [14] & $\sqrt{1 / \gamma}$ & $5.8 / 10$ & $1.6 / 10$ & $<0.01$ \\
\hline Feler [15] & $10 /$ SNS & $9.1 / 10$ & $4 / 10$ & NR \\
\hline Ghazwani [16] & 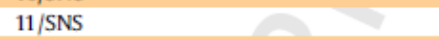 & $8.09 / 10$ & $5 / 10$ & $<0.001$ \\
\hline Heinze [43] & 20/PNM & $85 \mathrm{~mm}$ & $40 \mathrm{~mm}$ & 0.018 \\
\hline Kim [26] & 25 & $8.1 / 10$ & $4.1 / 10$ & $<0.01$ \\
\hline Maher [17] & $15 /$ SNS & $8.9 / 10$ & $2.4 / 10$ & $<0.001$ \\
\hline Marinkovic [18] & 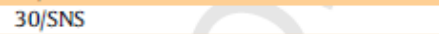 & $6.51 / 10$ & $2.43 / 10$ & \\
\hline \multirow{2}{*}{ Mira [35] } & 11/acupuncture-like TENS & $5.73 / 10$ & $2.55 / 10$ & 0.002 \\
\hline & $11 /$ TENS & $5.95 / 10$ & $2.48 / 10$ & $<0.001$ \\
\hline De Oliveira [41] & $24 /$ IES & $8.3 / 10$ & $2.1 / 10$ & $<0.05$ \\
\hline Ragab [27] & 20/PNS & $5.65 / 10$ & $5.25 / 10$ & NS \\
\hline Schneider [36] & 60/TENS & $6.6 / 10$ & $3.9 / 10$ & $<0.001$ \\
\hline Schiotz [37] & 21/TENS & $6.73 / 10$ & $5.18 / 10$ & 0.0009 \\
\hline Siegel [21] & $10 /$ SNS & $4.7 / 5$ & $2.2 / 5$ & NR \\
\hline Thomas [38] & 12 /TENS & $375 / 900$ & $245 / 353^{a}$ & $<0.05 / \mathrm{NS}$ \\
\hline \multirow[t]{2}{*}{ Tugay [39] } & $15 / \mathrm{EA}$ & $72.2 / 100$ & $17.5 / 100$ & $<0.05$ \\
\hline & 17/TENS & $79.4 / 100$ & $21.2 / 100$ & $<0.05$ \\
\hline \multirow[t]{2}{*}{ Thomas [38] } & 12 /TENS & $375 / 900$ & $245 / 353^{a}$ & $<0.05 / \mathrm{NS}$ \\
\hline & 19/acupuncture [26] & $412 / 900$ & $280 / 210^{a}$ & $<0.05 /<0.01$ \\
\hline Vallinga $[40]$ & 39/TENS & 8 & 3.2 & $<0.01$ \\
\hline Van Balken [28] & 33/PNS & 6.5 & 5.4 & $<0.05$ \\
\hline \multirow[t]{2}{*}{ Yang [48] } & 23/EMS & 5.5 & 3 & $<0.001$ \\
\hline & 22/ES plus biofeedback & 5.9 & 2.4 & $<0.01$ \\
\hline
\end{tabular}

EA = electrical acupuncture; EMS = electromagnetic stimulation; ES=electrical stimulation; IES=intravaginal electrical stimulation; SNS=sacral nerve stimulation; $\mathrm{SCS}=$ spinal cord stimulation; high freq EA = high-frequency electrical acupuncture; high freq MA =high-frequency manual acupuncture; low freq EA = low-frequency electrical acupuncture; low freq MA = low-frequency manual acupuncture; NR = not recorded; NRS= Numeric Rating Scale; NS=not significant; PNM = pudendal neuromodulation; PNS=percutaneous tibial nerve stimulation; RCT= randomized controlled trial; TENS=transcutaneous electrical nerve stimulation. 


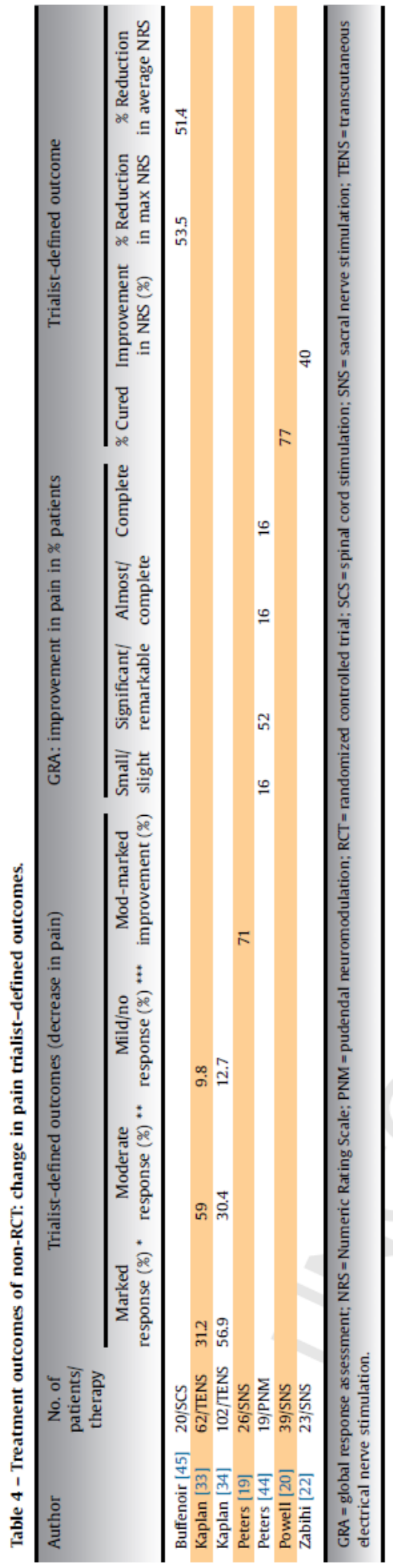



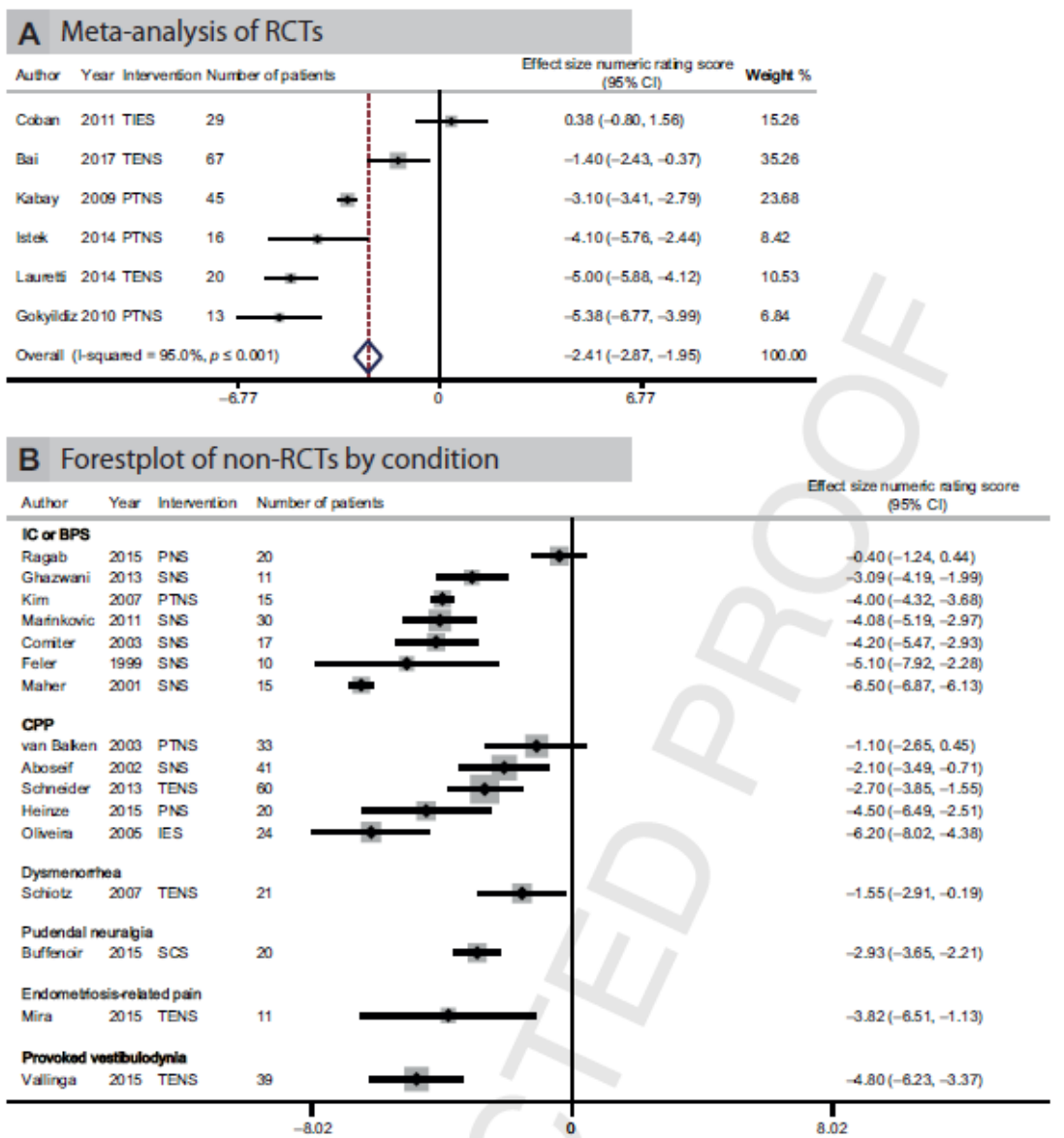

Forestplot on non-RCTs by treatment

\begin{tabular}{|c|c|c|c|c|}
\hline Author & Year & Intevertion & Number of patents & $\begin{array}{l}\text { Effect size numesic rafing scove } \\
\qquad(95 \% \mathrm{Cl})\end{array}$ \\
\hline \multicolumn{5}{|l|}{ PNS } \\
\hline Ragab & 2015 & PNS & 20 & $-0.40(-1.24,0.44)$ \\
\hline Heinze & 2015 & PNS & 20 & $-4.50(-6.49,-2.51)$ \\
\hline \multicolumn{5}{|l|}{ PTNS } \\
\hline van Baken & 2003 & PTNS & 33 & $-1.10(-2.65,0.45)$ \\
\hline Kim & 2007 & PTNS & 15 & $-4.00(-4.32,-3.68)$ \\
\hline \multicolumn{5}{|l|}{ TENS } \\
\hline Sctiotz & 2007 & TENS & 21 & $-1.55(-2.91,-0.19)$ \\
\hline Scineider & 2013 & TENS & $\infty$ & $-2.70(-3.85,-1.55)$ \\
\hline Mira & 2015 & TENS & 11 & $-3.82(-6.51,-1.13)$ \\
\hline Vallinga & 2015 & TENS & 39 & $-4.80(-6.23,-3.37)$ \\
\hline \multicolumn{5}{|l|}{ SNS } \\
\hline Abosolf & 2002 & SNS & 41 & $-2.10(-3.49,-0.71)$ \\
\hline Gharwani & 2013 & SNS & 11 & $-3.09(-4.19,-1.99)$ \\
\hline Mafinkovic & 2011 & SNS & 30 & $-4.08(-5.19,-2.97)$ \\
\hline Corriter & 2003 & SNS & 17 & $-4.20(-5.47,-2.93)$ \\
\hline Feler & 1999 & SNS & & $-5.10(-7.92,-2.28)$ \\
\hline Maher & 2001 & sNS & 15 & $-6.50(-6.87,-6.13)$ \\
\hline \\
\hline Buffenair & 2015 & $\operatorname{ses}$ & 20 & $-2.93(-3.65,-2.21)$ \\
\hline \multicolumn{5}{|l|}{ IES } \\
\hline Olveina & 2005 & IES & 24 & $-6.20(-8.02,-4.38)$ \\
\hline
\end{tabular}

Fig. 2 - Pain scores in RCTs and non-RCTs. (A) Meta-analysis of the difference in pain scores between treatment groups and control in RCTs. For Fig. 2 - Pain scores in RCTs and non-RCTs. (A) Meta-analysis of the difference in pain scores between treatment groups and control in RCTs. For
non-RCTs, forest plots of change in pain scores before and after the procedure by (B) condition and (C) treatment. BPS=bladder pain syndrome; $\mathrm{Cl}=$ confidence interval; $\mathrm{CPP}=$ chronic pelvic pain; IC = interstitial cystitis; IES = intravaginal electrical stimulation; PNS = pudendal nerve stimulation; PTNS = percutaneous tibial nerve stimulation; $R C T=$ randomized controlled trial; SCS = spinal cord stimulation; SNS = sacral nerve stimulation; TENS = transcutaneous electrical nerve stimulation; TIES = transcutaneous interferential electrical stimulation. 